\title{
Constat et analyse des politiques industrielles et scientifiques dans le contexte de l'intégration régionale du Sénégal
}

\section{Assessment and analysis of industrial and scientific policies in the}

\author{
context of Senegal's regional integration
}

\author{
Vanessa Casadella ${ }^{1}$, Sofiane Tahi $^{2}$ \\ ${ }^{1}$ Université Picardie Jules Verne, Réseau de Recherche sur l'Innovation, France, vanessa.casadella@u-picardie.fr \\ ${ }^{2}$ Université Picardie Jules Verne, France, Réseau de Recherche sur l'Innovation, sofiane.tahi@u-picardie.fr
}

RÉSUMÉ. Les politiques d'innovation reposent sur la Science et Technologie (S\&T), mais aussi sur des politiques indirectes permettant d'améliorer le contexte socio-économique local dans le cadre de Pays Moins Avancés (PMA). Notre article propose de valoriser les politiques industrielles et de S\&T dans le cadre de l'intégration régionale au Sénégal. La question soulevée est de savoir si le contexte de régionalisation du Sénégal dans le cadre de l'Union Economique et Monétaire Ouest-africaine (UEMOA) et la Communauté Economique des Etats de l'Afrique de l'Ouest (CEDEAO) lui est favorable, par opposition aux politiques protectionnistes marquées par I'Industrialisation par Substitution aux Importations (ISI) et pratiquée pendant des années. Dans un pays où la Science et Technologie (S\&T) sont principalement issues de la recherche scientifique agricole, notre proposition, mobilisant des données secondaires, met en lumière les différents enjeux du secteur industriel sénégalais dans un contexte porté par les politiques scientifiques et technologiques et la régionalisation grandissante.

ABSTRACT. Innovation policies are based on Science and Technology, but also on indirect policies to improve the local socio-economic context, in the context of Least Developed Countries (LDCs). Our article proposes to promote industrial and innovation policies within the framework of regional integration in Senegal. The question raised is whether the context of regionalization of Senegal within the framework of the West African Economic and Monetary Union (UEMOA) and the Economic Community of West African States (ECOWAS) is favorable to this, as opposed to the protectionist policies marked by Industrialization by Import Substitution (ISI) and practiced for years. In a country where Science and Technology are mainly focused on agricultural research, our proposal, mobilizing secondary data, highlights the various challenges of the Senegalese industrial sector in a context of science and technology policies and growing regionalization.

MOTS-CLÉS. Science, Technologie, Politique scientifique et technologique, Sénégal, Intégration régionale.

KEYWORDS. Science, Technology, Science and technology policies, Senegal, Regional integration.

Les politiques scientifiques et techniques émergent des travaux sur les systèmes d'innovation dans les pays industriels [FRE, 87]. Elles ont ensuite été appliquées dans les pays en développement pour actionner de nouveaux leviers de croissance économique fondés sur le rattrapage technologique et une meilleure mobilisation de la science. Un axe central dans le contexte de pays faiblement industriels, aux structures intersectorielles peu intégrées est focalisé sur les apprentissages et la construction de compétences comme objectif de réduction de la pauvreté [MUC, 03]. Plus largement, ces politiques dépendent aussi et surtout de l'environnement industriel et régional dans lequel elles baignent afin qu'elles puissent se construire et se développer à long terme.

Deux formes de politiques industrielles s'opposent traditionnellement : un cadre protectionniste, marqué par l'industrialisation par substitution aux importations (ISI), modèle de développement " autocentré » qui consiste à réduire la part des importations afin de développer le potentiel industriel d'un pays, et un cadre plus ouvert, libéral, marqué par la régionalisation, qui, dans le cas de l'Afrique Sub-Saharienne, s'associe à l'Union Economique et Monétaire Ouest-africaine (UEMOA) et la Communauté Economique des Etats de l'Afrique de l'Ouest (CEDEAO). Dans la pratique, ces 
politiques ne s'opposent pas, mais ne s'articulent pas toujours aisément dans un contexte économique et social complexe.

Nous souhaitons ici comprendre si le contexte de régionalisation du Sénégal a été bénéfique dans le cadre de politiques industrielles et scientifiques locales. En quoi la CEDEAO ou l'UEMOA ont elles permis à l'économie industrielle sénégalaise de construire des compétences scientifiques et techniques? Comment sont-elles complétées par les politiques locales ? Par la mobilisation de données secondaires, et des exemples de stratégies concrètes, nous montrons que, malgré les déficiences de la structure industrielle sénégalaise, le développement de dispositifs régionaux lui est plutôt favorable, dans un pays où la $R \& D$ est faible et principalement agricole. Mais ces dispositifs n'ont de sens qu'avec la concrétisation de mesures locales fortes et orientées vers l'industrie et la S\&T [BAS, 17].

Le choix du Sénégal est lié à la dynamique de son économie au sein de l'Afrique francophone et à la rareté des travaux liant les enjeux autour de la S\&T, du secteur industriel et du contexte régional. A ce titre, nous présentons d'abord une revue de littérature sur les politiques industrielles et liées à la Science et Technologie (S\&T) dans les Pays Moins Avancés (PMA) (1), puis nous opposons dans le cas du Sénégal, l'intégration régionale aux stratégies protectionnistes de l'ISI (2), et nous en déduisons enfin quel est le cadre le plus approprié pour favoriser une dynamique scientifique et technique et la construction de compétences technologiques au Sénégal (3).

\section{Politiques industrielles et de S\&T : Etat de l'art et implémentation du Sénégal}

\subsection{Conceptualisation autour des politiques de S\&T et difficultés de mise en place}

Si les politiques de S\&T sont un réel levier de croissance dans les pays industriels [BOR, 13], leur usage pour les politiques publiques des économies en développement est plus rare [DOG,09]. La littérature dans ce contexte identifie plusieurs évolutions du domaine de recherche qu'on qualifie de «S\&T ». Lundvall et Borras [Lun, 05] soulignent en quoi les politiques de S\&T sont nécessaires pour articuler une mise en système des domaines de la science et la technologie. Il n'en demeure pas moins que cette articulation est problématique dans certaines réalités empiriques d'économies en développement. Sur le plan conceptuel, les politiques scientifiques sont souvent orientées vers la création de connaissances académiques et la formation de scientifiques ou la recherche fondamentale [MIN, 18]. Les politiques technologiques sont davantage liées à la commercialisation ou la valorisation non académique des connaissances scientifiques.

Malgré ces différences de terminologies, les politiques S\&T restent un objet de recherche largement étudié [MAR, 12]. Elles se différencient néanmoins en fonction de la conceptualisation de l'innovation : large ou étroite [EDQ, 04]. Les premières concernent les politiques technologiques inhérentes aux infrastructures et au développement technologique promouvant la R\&D. Les secondes prennent en considération la formation du capital humain par l'éducation, l'enseignement supérieur, la formation professionnelle incluant également le conseil privé en entreprise [GOU, 15]. En soit, elles interpellent l'existence et la fonctionnalité dans l'innovation d'un ensemble complexe d'acteurs (publics, privés) que l'on peut qualifier de "services supports » à l'innovation [FAU, 19]. Elles réhabilitent parfois la contribution de travaux plus anciens sur la notion de capacités d'apprentissage [CRO, 97].

Afin d'identifier les instruments politiques les plus à mêmes de répondre au besoin de S\&T dans le contexte des PMA, il est nécessaire de comprendre la nature de l'innovation de ces derniers [CAS, 15]. En effet, l'hétérogénéité des systèmes d'innovation s'identifie autour des principaux problèmes suivants [CHA, 18]:

- Problèmes du manque d'infrastructures liées à des capacités d'investissements publics amoindries dans les infrastructures de R\&D (contexte d'émergence des Etats et des régulations 
publiques): capacité d'expérimentation et d'analyse, connectivité numérique, systèmes d'informations),

- Problèmes de la faiblesse des capacités technologiques d'absorption liées aux structures productives (faiblesses industrielles et des marchés),

- Problèmes d'informalité et d'interconnectivité des réseaux sociotechniques,

- Problèmes institutionnels liés à la faible fonctionnalité des institutions (ou leur défaillance dans le cas de corruption) qui accompagnent les processus d'innovation (évaluation et prise en compte des risques), les orientant (subventions réglementation, fiscalité) ou les gouvernant,

- Problèmes de transition et de lock-in liés aux caractéristiques socio-historiques des dépendances liées à l'histoire coloniale, au développement des entreprises globalisées, aux régimes politiques rentiers ou aux aversions diverses aux progrès scientifiques et techniques (créationnisme...).

L'ensemble des difficultés institutionnelles et infrastructurelles sont des freins aux dynamiques technologiques. Il est néanmoins à souligner que depuis les indépendances, de nombreux pays en développement et moins avancés ont investi dans un certain nombre de ressources (enseignement supérieur, institutions nationales de recherche) dont l'efficacité peut encore être encore renforcée par une meilleure gouvernance systémique [BEN, 17]. Les difficultés (ou les impossibilités) à disposer de mesures actionnables pour évaluer des processus systémiques complexes et donc de renseigner l'action des politiques publiques sont un facteur limitant de cette gouvernance.

Pour définir une politique scientifique cohérente, il est nécessaire de comprendre quels sont les problèmes prioritaires au regard des contraintes précitées et identifiées dans le système d'innovation [BOR, 13]. Ces problèmes sont définis comme la faible capacité du système d'innovation à soutenir la création, l'absorption, l'utilisation et la diffusion de connaissances grâce à l'apprentissage interne interactif ou en R\&D [CAR, 97]. Il est dès lors légitime de connaître les causes de ces défaillances afin d'être en mesure de choisir les instruments politiques appropriés. L'idée est de ne pas retenir les instruments « optimaux » mais de considérer un certain nombre d'instruments en fonction de leur contexte. Dans cette perspective, les gouvernements sont censés pouvoir élaborer des politiques d'innovation intégratives face aux problèmes systémiques spécifiques pour construire des systèmes nationaux d'innovation pérennes.

\subsection{Politiques industrielles et de S\&T au Sénégal}

D’après le Centre Unique de Collecte de l'Information (CUCI), le Sénégal compte en 20201947 entreprises industrielles composées à 97,3\% de Petites et Moyennes Industries (PMI) et de 2,7\% de grandes entreprises industrielles. Néanmoins, le secteur industriel ne représente que $18 \%$ de la population active et ne contribue que pauvrement à la croissance économique. Non seulement le capital est presque entièrement détenu par des investisseurs étrangers (suite à des problèmes de gouvernance ou de mouvements syndicaux), mais la plupart des sénégalais pouvant accéder aux sources modernes de financement préfèrent investir dans le commerce.

Comme le montre le tableau ci-dessous, les secteurs les plus représentatifs sont l'agroalimentaire, la mécanique et métallurgie, le bois, papier, carton et imprimerie, la chimie et le plastique, l'énergie, les industries extractives, le textile, habillement, cuirs et peaux. La création de richesses émane surtout des secteurs liés à l'agroalimentaire, chimie et plastique et énergie.

De façon générale, le secteur industriel est surtout mis en difficulté par les problèmes liés aux conditions d'exploitation et de productivité. Le pays est passé d'une « économie à dominante agraire à une économie tertiaire en sautant l'étape de l'industrialisation » [FMI,17]. De plus, il existe une forte dualité dans ce secteur entre le formel et l'informel lequel rassemble de grands importateurs. Enfin, on recense plus globalement un manque de compétitivité sur les facteurs de production et un 
accès difficile et onéreux au crédit. Au final, l'industrie est soumise à deux contraintes majeures : les coûts élevés des facteurs de production et la concurrence déloyale du secteur informel (fraude, sousfacturation et dumping) [MAN, 18].

\begin{tabular}{|c|c|}
\hline Secteur & $\begin{array}{c}\text { Part représentative du secteur } \\
(\mathbf{2 0 1 7})\end{array}$ \\
\hline Agroalimentaire & $47,8 \%$ \\
\hline Mécanique et métallurgie & $12,2 \%$ \\
\hline Bois, papier, carton, imprimerie & $11,2 \%$ \\
\hline Chimie, Plastique & $8,5 \%$ \\
\hline Energie & $5,7 \%$ \\
\hline Industrie extractive & $6,3 \%$ \\
\hline Textile, habillement, cuirs et & $3,5 \%$ \\
\hline peaux & $4,8 \%$ \\
\hline Autres industries & \\
\hline
\end{tabular}

Source : informations issues de www.ansd.sn

Tableau 1. Part des principaux secteurs industriels du Sénégal

Concernant l'émergence d'une S\&T dans le secteur industriel, force est de constater que la S\&T tirée par la R\&D peine à émerger dans ce secteur : non seulement la R\&D reste principalement agricole, mais elle ne dépasse pas 0,89\% des dépenses publiques [AST, 18]. Les liens entre le secteur industriel et académique ne sont ni structurés ni formalisés. Les systèmes d'innovation et technologiques sont décrits comme défaillants, notamment en raison du caractère déconnecté entre le système éducatif et productif [CAS, 17]. Sans revenir sur les indicateurs output de l'innovation, la politique de $\mathrm{S} \& \mathrm{~T}$ est fragile, et très peu représentative dans le secteur industriel (aucune donnée n'est disponible pour la $R \& D$ industrielle, visiblement peu contributive de la R\&D totale). Il va donc falloir compter sur les politiques indirestes, telles que les principales réformes liées aux politiques fiscales, d'apprentissage ou commerciales. Le Plan Sénégal Emergent (PSE) ouvre en ce sens de nouvelles perspectives d'industrialisation en améliorant l'écosystème physique dans lequel les industries évoluent [MAN, 18].

L'objectif général du PSE est de porter la contribution du secteur à la croissance du PIB à au moins $25 \%$ en 2025 et de créer des emplois massifs [GOU, 18]. Pour ce faire, les principales stratégies mises en place sont l'amélioration de la gestion du secteur à travers des réformes légales et institutionnelles, le renforcement de la compétitivité industrielle, la valorisation des ressources locales, une répartition équilibrée du tissu industriel et le financement durable du secteur. Quatre projets ont été retenus dans le PSE: la mise en place de plateformes industrielles intégrées, les agropoles compétitifs, les parcs industriels et hub minier régional. Ces projets ou objectifs sont à encourager dans un pays où le secteur industriel n'est pas une priorité nationale, au même titre que l'agriculture. Mais l'émergence du secteur industriel reste peu concrète, qui plus est actuellement, dans un contexte de crise sanitaire, ayant mis à mal une grande majorité d'entreprises formelles.

\section{Intégration régionale vs. stratégies d'Industrialisation par Substitution aux Importations (ISI) au Sénégal}

L'ouverture régionale a été reconnue comme l'une des possibilités permettant aux entreprises de moderniser leurs activités de production par l'accès aux biens et technologies des pays les plus avancés. Elle permet également l'expansion de la demande des biens locaux et l'augmentation de la 
productivité, puisque l'exposition à la concurrence internationale oblige les entreprises à être plus efficaces et innovantes. Mais le Sénégal a également été précurseur en matière d'Industrialisation par Substitution aux Importations (ISI) dès les années 1960 pour protéger son industrie locale.

\subsection{L'ISI : gain ou perte de compétitivité pour l'économie locale?}

L'Industrialisation par Substitution aux Importations (ISI) est un modèle de développement économique «autocentré » qui consiste à réduire la part des importations afin de développer le potentiel industriel d'un pays. En limitant les importations, l'industrie du pays se développe pour répondre à la demande interne. Cela permet de protéger l'industrie locale et le développement d'entreprises étrangères trop robustes. Son objectif premier est de garantir l'autonomie économique nationale par la création d'une capacité industrielle de substitution aux importations et de transformation pour l'exportation hors du continent des produits agricoles et miniers [BIK, 10].

À la suite de son accession à l'indépendance le 20 juin 1960, le Sénégal, comme bon nombre de pays en développement, s'est orienté vers une politique d'ISI. Trois arguments ont été mis en exergue pour justifier l'intervention permanente de l'Etat [JAC, 93] :

- les retards et les handicaps structurels justifiant le prix d'un effort de l'Etat, à la fois conscient, massif et dirigé ;

- la mise en œuvre de grands projets technologiques, vecteurs de l'indépendance ;

- la concentration autour de quelques pôles de croissance géographiques et sectoriels, vecteurs de croissance en économie ouverte.

Tout en acceptant le capital étranger, le Sénégal se lance donc dans une politique tournée vers l'intérieur. Ainsi, l'ISI est instituée, avec le développement par l'Etat d'une industrie de transformation des produits agricoles, huileries essentiellement. Des monopoles publics sont alors observés dans les huileries et les industries liées au coton et au riz. Mais les résultats de l'ISI sont marqués par un large échec, illustré notamment par la compétitivité déclinante de l'économie sénégalaise. En effet, cette politique a eu des effets négatifs sur les exportations du pays, sans pour autant permettre au gouvernement de collecter davantage de recettes tarifaires [ANN, 05]. De même, les importations n'ont cessé d'augmenter en dépit de leur objectif premier visant à diminuer progressivement voire à remplacer les dépenses d'importation et à retrouver l'équilibre de la balance commerciale. les importations ont commencé à exploser à partir de 1977 et des déséquilibres économiques, financiers et monétaires sont apparus, conduisant à des réformes structurelles, compatibles avec une politique de libéralisme économique. Les instruments de politique économique préconisés par le Fond Monétaire International et la Banque Mondiale ont alors ambitionné d'assurer la flexibilité de l'économie, à limiter les rentes et à faire émerger les acteurs innovants, par deux voies : la stabilisation et l'ajustement. Néanmoins, le recours au capital étranger, la politique tournée vers l'intérieur et l'ISI, restent en vigueur. Mais dans la décennie 1990, le modèle de l'ISI a été source de rigidités structurelles, liées à l'absence de concurrence (oligopoles ou monopoles) et à une rentabilité axée sur la rente (et non le profit). Si l'on tient compte de la faiblesse des emplois créés, de l'existence de larges surcapacités de production, le modèle a débouché sur une caricature de «stagflation» [OMA, 94].

Dès lors, et à partir de 1986, le Sénégal privilégie l'ouverture sur l'extérieur en cherchant à développer et à diversifier ses exportations. C'est dans ce contexte que le Sénégal a élaboré et mis en œuvre la nouvelle politique industrielle (NPI) initiée en février 1986, la politique de redéploiement industriel (PRI) en juillet 2004 puis d'autres variantes de la PRI. Ces politiques n'ont, ni réduit les importations, ni augmenté les exportations. Elles ont abouti à l'échec des privatisations et à des coûts comparatifs élevés du Sénégal faute d'insertion nette dans la division internationale du travail et de choix pertinents d'industrialisation. Enfin, un PSE (Plan Sénégal Emergent), a été mis 
en œuvre depuis 2014. Ce plan, marqué par une nouvelle politique industrielle est axé sur l'ouverture, avec une nette insertion vers l'économie mondiale et un recours au capital national et étranger ainsi qu'aux investissements publics.

Le Sénégal est donc passé d'un modèle d'ISI peu concluant, à un PSE, plus libéral, orienté vers le développement d'hubs logistiques et industriels, l'encouragement à l'innovation ou l'exploitation de la propriété industrielle. Cette réorientation radicale démontre que la stratégie de l'ISI n'est pas payante dans ce pays et qu'il a fallu repenser l'industrialisation de pair avec le contexte mondial actuel. Il est clair que portée à la problématique africaine globale, l'ISI a été décriée car elle n'a ni réduit ses importations, ni rendu la production du continent compétitive, ni encore moins créé un véritable tissu industriel ; elle a plutôt aggravé les tensions sociales et a déclenché un véritable mouvement de désindustrialisation [BIK, 10].

\subsection{Stratégies d'industrialisation et régionalisation}

Depuis les indépendances, l'intégration régionale en Afrique de l'Ouest a été marquée par de multiples initiatives. La CEDEAO et l'UEMOA sont deux organisations à vocation économique qui œuvrent chacune selon ses objectifs à la réalisation de cette intégration économique. Les huit pays de l'UEMOA, dont font partie le Bénin, le Burkina Faso et le Mali, sont également membres d'un ensemble plus vaste de quinze pays : la CEDEAO. L'intégration au sein de la CEDEAO s'inspire largement de celle de l'UEMOA. Même si elles traduisent un enthousiasme politique certain, leurs effets ont été limités compte tenu des faibles capacités organisationnelles et des structures économiques peu adaptées à une intensification des échanges régionaux [HUG, 01]. Néanmoins, une nouvelle dynamique émerge avec l'évolution de l'UEMOA qui, en moins de cinq ans, est venue à terme de son union douanière, avec la mise en place d'un système de surveillance multilatérale des politiques macro-économiques.

Si globalement les mécanismes de libéralisation des échanges sont correctement appliqués par les États, un certain nombre de difficultés est relevé. Elles sont liées notamment aux barrières non tarifaires rendant difficile la fluidité des échanges, à la faible sensibilisation du public et des opérateurs économiques, aux faibles capacités statistiques commerciales et financières, au poids des échanges informels et à la fragilité socio-politique des pays membres qui remet en cause le respect des critères de convergence [HUG, 01]. Sur le plan industriel, les pays de l'UEMOA ont adopté en décembre 1999, une Politique Industrielle Commune (PIC) pour le besoin de la restructuration des unités industrielles, la promotion d'un tissu industriel coordonné, l'amélioration de la compétitivité des entreprises et le développement industriel durable. Les objectifs fixés sont notamment l'accélération et l'adaptation de l'industrie de l'Union aux changements structurels en cours ou la promotion de la mise en place d'un environnement favorable à l'initiative privée. Cette politique a contribué à l'insertion des économies des membres au processus de mondialisation en donnant naissance à un Tarif Extérieur Commun (TEC) et à une Politique Commerciale Commune (PCC).

Quant à la CEDEAO, son programme de libéralisation des échanges a beaucoup moins avancé. Sur sa politique industrielle, bien que de nombreuses initiatives aient été lancées, chaque Etat membre a poursuivi la mise en œuvre de sa propre politique d'industrialisation. Cela s'est traduit par une quasi-absence de collaboration à l'échelle régionale, ne permettant pas d'atteindre les objectifs attendus en la matière. Face à la situation du secteur industriel de l'Afrique de l'ouest, la CEDEAO a élaboré la mise en œuvre de la vision 2020 adoptée en 2007, et créé la Politique Industrielle Commune de l'Afrique de l'Ouest (PICAO). Les objectifs généraux de la Politique Industrielle Commune de l'Afrique de l'Ouest (PICAO) consistent à œuvrer en vue de l'accélération de l'industrialisation de l'Afrique de l'Ouest par le biais de la promotion de la transformation industrielle endogène des matières premières locales, le développement et la diversification des capacités productives industrielles, et le renforcement de l'intégration régionale et des exportations de biens manufacturés. Les stratégies de la PICAO mettent en particulier l'accent sur le renforcement du commerce intra régional. Cela implique de supprimer les barrières tarifaires et non 
tarifaires et d'harmoniser les incitations aux investissements pour encourager un développement industriel équitable dans la région. De ces deux cadres institutionnels autant complémentaires que concurrents, en ressortent une volonté commune de promouvoir la dynamique industrielle, tout comme un manque d'interdépendance économique pour avoir un impact réel sur la croissance locale. Le secteur industriel dans la plupart des pays de la région demeure embryonnaire et n'a pas toujours été mis à niveau.

\section{Politiques industrielles et de S\&T au Sénégal : quel cadre approprié ?}

Dans le cas du Sénégal, deux projets montrent l'importance de l'ouverture du pays dans une optique de promotion de l'industrie et de la S\&T: la législation des brevets, et les différents dispositifs incitatifs à l'innovation. Il est toutefois essentiel de rendre cohérents ces projets à d'autres réformes locales, qui, sans aller, vers un cadre protectionniste excessif, vise simplement à mettre en parallèle ouverture du secteur industriel et adéquation de ce secteur avec le marché national.

\subsection{L'influence du cadre régional sur les politiques industrielles et de S\&T}

\section{L'exemple du droit de la Propriété Intellectuelle}

Un des points essentiels pour la mise en place d'une dynamique innovante au sein du secteur industriel est la protection des droits de la propriété intellectuelle. L'Organisation Africaine de la Propriété Intellectuelle (OAPI) des Etats membres de l'UEMOA est chargée de mettre en œuvre et d'appliquer les procédures administratives communes découlant du régime uniforme de protection de la propriété industrielle. C'est elle qui centralise toutes les demandes de protection des droits de propriété industrielle, les instruit et délivre les titres de protection. Elle perçoit les taxes de dépôt et de maintien en vigueur des titres. L'Organisation est également chargée de promouvoir le développement des Etats membres au moyen d'une protection efficace de la propriété intellectuelle et des droits connexes. Sa branche au Sénégal est l'Agence Sénégalaise de la Propriété Industrielle et de l'Innovation (ASPIT). Cette Agence est particulièrement importante, car, grâce au soutien de l'OAPI, elle coordonne les actions entre les acteurs institutionnels, sensibilise les chercheurs des milieux académiques dans la mise en œuvre de recherches collaboratives dans une logique d'open innovation et impulse de nouveaux partenariats en vue de développer l'innovation. En subventionnant des projets innovants, elle met en synergie des acteurs locaux pour le renforcement des capacités innovantes du pays. Le système de protection des droits de propriété intellectuelle dans les pays membres de l'UEMOA veille également à assurer la promotion de la propriété industrielle. Cette protection agit dans le cadre de la mise en réseau des services de propriété intellectuelle. En ce sens, le Sénégal a inscrit dans ses documents de politique de développement économique la promotion de l'intelligence économique et de la propriété industrielle. S'il ne se classe que 102ème sur 129 pays dans le Global Innovation Index de 2020, il est positionné 7ème pays d'Afrique subsaharienne, avec de bons résultats dans le domaine des Technologies d'Information et de Communication. En matière de brevets, les dépôts sont très limités (20 demandes déposées en 2018) et les marques sont principalement déposées par des sociétés étrangères (données OMPI).

\section{Les dispositifs régionaux commerciaux et technologiques}

Les dispositifs incitatifs issus de la régionalisation ne sont pas toujours directement liés à la S\&T mais ont leur importance pour promouvoir le secteur industriel dans une région où la compétitivité fait défaut et dans un contexte international en renouvellement permanent. Sur le plan commercial, les biens produits dans la région sous licence du dispositif de libéralisation des échanges CEDEAO bénéficient à la fois d'une suppression des droits de douane et d'une protection tarifaire allant jusqu'à $35 \%$ à l'égard de ceux originaires de pays tiers importés dans la CEDEAO. Moins un 
produit bénéficie de transformation, moins il est taxé. La taxation par droits de douane varie entre 0 $\%$ pour les biens de consommation courante et $20 \%$ pour les produits finis destinés à la consommation. Les produits lourdement taxés (35\%) sont des biens destinés spécifiquement au développement économique, l'objectif étant de protéger les filières locales de production. Du côté de l'UEMOA, c'est le dispositif PIC (Politique Industrielle Commune) qui permet de promouvoir les investissements et les exportations, le développement des PMI et des réseaux d'informations. Grâce à l'accélération de réformes dans certains secteurs stratégiques, un environnement propice à l'instauration du secteur privé peut être encouragé.

Sur le plan de l'activité technologique, la mise en place de l'Elaboration de la Politique de la CEDEAO sur la Science, la Technologie et l'innovation (ECOPOST) permet aux Etats membres de parvenir à un développement économique et social durable pour satisfaire les besoins actuels et leur garantir une meilleure qualité de vie. Parmi ses missions, on retrouve, entre autres, la planification et la coordination des programmes et activités en matière de Recherche Scientifique et Innovation, la définition de la stratégie régionale en matière de science et d'innovation, la promotion des activités scientifiques nécessaires à la diffusion de l'innovation entre les universités, les instituts de recherche et les entreprises ou la mise en place de mesures incitatives (primes, prix, distinctions honorifiques, bourses, facilités de voyages d'étude etc..) pour motiver et encourager les chercheurs méritants. Selon l'UNESCO [UNE, 15] cette politique fournit un cadre aux États membres qui souhaitent améliorer leurs propres politiques et plans d'action nationaux en matière de $\mathrm{S} \& \mathrm{~T}$ et comprend un mécanisme de suivi et d'évaluation de la mise en œuvre des politiques. Elle aborde également la question du financement, en proposant la création d'un fonds de solidarité géré par un directoire relevant de la CEDEAO afin d'aider les pays à investir dans des institutions clés et à améliorer l'éducation et la formation. Plus globalement, cette politique régionale préconise le développement d'une culture scientifique dans tous les secteurs de la société. Au total, et comme le montre le tableau ci-dessous, un certain nombre de dispositifs agissent positivement sur le secteur industriel et la promotion de la S\&T. Mais encore faut-il qu'il y ait une réelle cohérence entre la mise en place de ces dispositifs et les mesures entreprises localement.

\begin{tabular}{|c|c|c|}
\hline $\begin{array}{c}\text { Portée de la régionalisation } \\
\text { sur les politiques industrielles } \\
\text { et de S\&T }\end{array}$ & $\begin{array}{c}\text { Actions liées au } \\
\text { développement technologique }\end{array}$ & $\begin{array}{c}\text { Actions indirectement liées au } \\
\text { développement technologique }\end{array}$ \\
\hline CEDEAO & $\begin{array}{c}\text { Dispositif ECOPOST de soutien } \\
\text { et promotion de la S\&T }\end{array}$ & $\begin{array}{c}\text { Dispositif de libéralisation des } \\
\text { échanges de taxation par droits } \\
\text { de douane }\end{array}$ \\
\hline UEMOA & $\begin{array}{c}\text { Dispositif de protection des } \\
\text { droits de la propriété } \\
\text { intellectuelle (OAPI) pour les } \\
\text { pays membres de l'UEMOA }\end{array}$ & $\begin{array}{c}\text { Dispositif PIC : Politique } \\
\text { Industrielle Commune } \\
\text { (élaboration de programmes } \\
\text { communs, promotion des PMI, } \\
\text { réseaux d'informations) }\end{array}$ \\
\hline
\end{tabular}

Tableau 2. Portée de la régionalisation sur les politiques industrielles et de $S \& T$

\subsection{La protection de l'industrie locale et la création de compétences technologiques}

Les dispositifs régionaux d'aide à l'appui du secteur industriel et de S\&T ne doivent pas faire oublier l'intérêt pour un pays comme le Sénégal qui peine à faire émerger sa dynamique industrielle et surtout maintenir le cap fixé par le PSE. En cela, il reste nécessaire à la fois de protéger son industrie locale mais également de créer des compétences techniques locales favorables au secteur industriel. 


\section{La protection de l'industrie locale}

En 2019, la balance commerciale sénégalaise est déficitaire car le Sénégal est très fortement dépendant de certains biens qu'il ne produit pas et ne commercialise pas. Ainsi, en 2019, selon les données de l'OMC ${ }^{1}$, ses importations de biens représentent 8,143 millions de dollars, tandis que ses exportations, 4,175. Ses principaux fournisseurs sont la France, la Chine et la Belgique. Pour survivre, le pays étant très dépendant des importations, les activités industrielles, spécialisées dans les produits de première nécessité (farine, sucre, huile) doivent pouvoir se substituer intelligemment aux importations. De plus, si les réformes proposées par la CEDEAO et l'UEMOA visent à restructurer certains secteurs pour mieux libérer les initiatives privées, elles ont également favorisé une déstructuration du tissu productif et une désorganisation de l'architecture commerciale (notamment pour l'huilier SONACOS). Il faut également préciser que l'industrie sénégalaise doit faire face à la concurrence déloyale d'importations massives de produits (souvent d'origine asiatique) qui contournent la réglementation commerciale [MAN, 18]. Les mesures de protection de l'industrie locale s'avèrent donc incontournables. Ces mesures peuvent être des barrières tarifaires comme les droits de douane, en accord avec les règles de l'Organisation Mondiale du Commerce (OMC) et de UEMOA ou des barrières non tarifaires comme les quotas, les licences, ou les exigences en termes de certifications ou de conformité. Par exemple, l'interdiction d'importer des produits avicoles au Sénégal est en vigueur depuis 2005. Dans cette même direction, certaines taxes à l'importation et période de gel annuel dans certaines filières (agricoles comme l'oignon) et ont été particulièrement bénéfiques. L'État a également massivement subventionné les intrants pour booster la production de la filière rizicole. Pour la filière sucrière, les importations ont été autorisées pour combler la quantité de sucre que ne pourrait produire la Compagnie Sénégalaise de Sucre (CSS) détenant le monopole sur la filière, et ont mis fortement à mal l'emploi et le chiffre d'affaires de l'industrie. Mais pour cela, la pression est mise sur le prix du sucre, bien largement inférieur dans les pays limitrophes.

Si des mesures sectorielles ont été plus parlantes dans l'agro-industrie, il faut aller chercher dans les principales réformes économiques portées par le PSE et touchant à l'environnement économique des affaires pour y voir un réel effort de l'Etat pour dynamiser son secteur industriel. Comme rappelé en première partie, on peut citer la création de zones industrielles, la règlementation des affaires, le soutien de l'investissement ou la consolidation de la règlementation de la concurrence. Toutes ces mesures ont pour objectif de soutenir le secteur privé, sans toutefois réellement protéger son marché intérieur.

\section{Créer des compétences technologiques pour favoriser des synergies locales}

Encourager le secteur industriel et dynamiser la S\&T, c'est aussi et surtout renforcer les capacités nationales. Or, le système d'innovation et de recherche sénégalais est émietté avec une gouvernance globale complexe, peu claire et des infrastructures très nombreuses [CAS, 17].

\footnotetext{
${ }^{1}$ Selon les données disponibles sur www.wto.org
} 


\begin{tabular}{|c|}
\hline Sénégal - 2021 \\
\hline Education Supérieure \\
\hline Universités publiques: \\
\hline UCAD: Université Cheikh-Anta-Diop de Dakar \\
\hline UGB: Université Gaston-Berger, de Saint-Louis \\
\hline UADB: Université Alioune Diop de Bambey \\
\hline UT: Université de Thiès \\
\hline UASZ: Université Assane Seck de Ziguinchor \\
\hline UVS: Université Virtuelle du Sénégal \\
\hline USSEIN: University du Sine Saloum El Hadji Ibrahima Niass \\
\hline UAM: Université Amadou Mahtar Mbow \\
\hline Universités publiques: \\
\hline Université Amadou Hampaté Bâ Dakar-Bourguiba \\
\hline Université du Sahel \\
\hline Université Catholique de l'Afrique de l'Ouest \\
\hline Université Barma El Hadji Ibrahima Niass \\
\hline Institut de Technologie de Dakar \\
\hline Centres publics de recherche \\
\hline Institut sénégalais de recherche agricole \\
\hline Institut de technologie alimentaire \\
\hline Centre d'étude et de recherche sur les énergies renouvelables \\
\hline Institut national de pédologie \\
\hline Centre de surveillance écologique \\
\hline Centre de recherche en écotoxicologie du Sahel \\
\hline Laboratoire de la Direction de la protection des végétaux \\
\hline Agence nationale de la recherche scientifique appliquée \\
\hline Agence sénégalaise pour l'innovation technologique \\
\hline Centres de recherche étrangers, régionaux et internationaux (BREDA, CRDI, CRAT, IRD) \\
\hline Centres privés de recherche \\
\hline Fondation Pasteur \\
\hline Prometra \\
\hline Manobi \\
\hline Ceres locutox \\
\hline Sodefitex \\
\hline
\end{tabular}

\section{Graphique 1. Infrastructures de S\&T au Sénégal}

Si de nouvelles universités émergent (comme l'Université Virtuelle du Sénégal), la mise en place d'une politique d'apprentissage tout au long de la vie (formation professionnelle, formation en entreprise, congé de formation, etc.) est rendue difficile dans des contextes de forte concentration industrielle et de structure informelle dominante au sein d'un tissu de petites et microentreprises. Dès lors, l'approche systémique de la $\mathrm{S} \& \mathrm{~T}$ prend tout son sens dans ce pays avec la création d'institutions telles que l'ISADE (Institut Supérieur Africain pour le Développement de l'Entreprise) ou la réorientation du système d'enseignement supérieur vers la science, technologie et la formation professionnelle courte. Le gouvernement a donc créé à ce titre des Instituts Supérieurs d'Enseignement Professionnel (ISEP) dans toutes les régions du pays. Le projet pédagogique de tous les ISEP est basé sur l'approche par les compétences et conduit à un diplôme de niveau bac+2. Ces instituts sont importants pour le développement des apprentissages et des compétences. Ils permettent de créer davantage de demande de connaissances, dans un contexte de professionnalisation et d'acquisition rapide des connaissances. Le milieu académique était jusque-là particulièrement isolé du milieu industriel dans un pays où les principales sources d'apprentissage sont sur le tas et informelles [CAS, 10]. Par ailleurs, l'utilisation rapide et efficace des nouvelles technologies par une main-d'œuvre qualifiée nécessite des investissements dans la formation d'ingénieurs technologiques présents dans tous les secteurs, y compris industriels. A ce titre et depuis 2014, un nouveau fonds, le 3FPT soutient la formation des salariés en entreprises, des jeunes en recherche de qualification, des centres et institutions de formation mais aussi l'assurance qualité du système de formation professionnelle et technique. Ce fond est louable pour la modernisation des connaissances et apprentissages internes. Enfin, si le Sénégal a engagé de profondes réformes en faveur de l'enseignement supérieur et de sa professionnalisation, ce n'est pas seulement pour se tourner vers un marché du travail plus adapté mais aussi pour éviter une fuite massive des cerveaux. 
Chaque année, la migration des étudiants sénégalais est de plus en plus forte et concerne davantage une population qualifiée. Dans cette dimension, le gouvernement a agi bénéfiquement par la mise en place d'une Direction de l'Assistance et de la Promotion des Sénégalais de l'Extérieur et de programmes spécifiques d'assistance et d'encadrement de l'immigré qualifié.

L'ensemble des réformes, résumées ci-dessous et principalement issues du PSE, sont non seulement favorables à l'amélioration du secteur industriel, mais à son intégration réussie dans une économie internationale. Si elles ne contribuent pas toutes directement à l'amélioration de la S\&T toujours faible dans ce pays ou à la protection du marché intérieur (qui ne pourrait être porté que par des mesures sectorielles fortes), elles permettent d'améliorer sensiblement l'environnement industriel local.

\begin{tabular}{|c|c|c|c|}
\hline Orientation & Politique commerciale & $\begin{array}{c}\text { Politique fiscale et } \\
\text { industrielle }\end{array}$ & $\begin{array}{c}\text { Politique de } \\
\text { l'apprentissage }\end{array}$ \\
\hline Outils & $\begin{array}{c}\text { Mesures quantitatives et } \\
\text { non quantitatives de } \\
\text { taxation des produits } \\
\text { industriels }\end{array}$ & $\begin{array}{c}\text { Règlementations, parcs } \\
\text { industriels, taxation }\end{array}$ & $\begin{array}{c}\text { Nouvelles infrastructures } \\
\text { tournées vers le marché } \\
\text { du travail }\end{array}$ \\
\hline Exemples & $\begin{array}{c}\text { Protection de filières } \\
\text { industrielles par la mise } \\
\text { en place de taxes } \\
\text { d'importation sur } \\
\text { certains secteurs }\end{array}$ & $\begin{array}{c}\text { Amélioration de la } \\
\text { réglementation du droit de } \\
\text { la concurrence et de la } \\
\text { règlementation du droit } \\
\text { des affaires }\end{array}$ & $\begin{array}{c}\text { Création des ISEP } \\
\text { Rencement de l'outil } \\
\text { numérique }\end{array}$ \\
Assistance aux immigrés \\
qualifiés
\end{tabular}

Tableau 3. Principales réformes internes en faveur d'une dynamique industrielle et de S\&T

\section{Conclusion}

Les politiques scientifiques et techniques doivent être repensées autour de réponses appropriées aux enjeux futurs à pouvoir d'une part, s'adapter aux crises générées par les modèles de développement actuels, et d'autres part, saisir des opportunités de transformation qu'offrent les progrès de la science et de la technologie souvent concentrés dans les pays industriels [ROD, 10]. A la question de savoir si le cadre régional est propice au développement de la S\&T dans le secteur industriel au Sénégal, la réponse est positive mais nuancée dans une économie où ce secteur est peu représentatif de la dynamique économique nationale.

Le cadre régional au sein duquel le Sénégal est inséré n'est plus discutable. Il faut donc pouvoir bénéficier de ses forces et savoir se les réapproprier localement. Les dispositifs mis en place par l'UEMOA et la CEDEAO de promotion de l'industrie et l'innovation sont nombreux et salutaires. Néanmoins, au Sénégal, où l'industrie est marquée par le secteur agro-industriel, on dénonce encore une concurrence des importations trop élevée ou déloyale, soit du fait de la mise en place d'un tarif extérieur commun, soit de la zone de libre-échange UEMOA-CEDEAO, soit du fait de produits ne respectant pas les normes ou les règles d'origine [ALP, 10]. De plus, les pays membres ne se concertent pas toujours dans la mise en place d'actions communes, mettant à mal les dispositifs entrepris et pointant le manque de cohérence globale. Par ailleurs, les résultats ne sont pas au rendez-vous si l'on prend l'exemple des mesures d'output de la S\&T et le nombre de brevets déposés par le Sénégal (tous secteurs confondus).

Face à ce manque de cohérence, et de concurrence déloyale et de faibles résultats, il est impérieux que le Sénégal protège son industrie en valorisant ses capacités techniques. Mais ce protectionnisme doit être « intelligent », calibré et surtout savoir profiter aux populations locales. Pour cela, le pays 
est confronté à un double enjeu : soutenir son industrie tout en créant des capacités locales. Sur le soutien de l'industrie, le dilemme est réel puisqu'il faut à la fois protéger ses industries tout en s'alignant aux pressions du marché. La solution, très fortement appuyée par les recommandations des institutions internationales, consiste à implémenter des politiques sectorielles fortes pour encadrer toute forme de protectionnisme et le rendre approprié au secteur concerné [UNE, 15]. Ces politiques sectorielles sont parfois plus marquées que d'autres, selon les intérêts économiques qu'elles revêtent. Les Ministères doivent alors mieux collaborer pour planifier les importations et éviter des scénarios de mévente que les producteurs redoutent en raison de la forte présence de produits importés sur le marché sénégalais. Quoi qu'il en soit, l'ISI n'est plus adaptée à l'économie sénégalaise, et doit être remplacée par un protectionnisme dosé, temporaire et complété par la création de capacités technologiques industrielles locales.

Sur l'aspect de création de capacités, les différentes réformes entamées dans le PSE (sur les politiques d'apprentissage, commerciales, fiscales) sont largement appréciables (si maintenues à long terme) et montrent la volonté de l'Etat d'accompagner, à défaut de mise en place de réelles politiques de S\&T, le secteur industriel local. Ces réformes sont également appropriées eu égard aux mesures entamées sur le plan régional. Néanmoins, l'industrie reste balbutiante, encore peu affirmée, alors que le Sénégal a le potentiel pour répondre aux attentes des consommateurs, notamment dans le secteur agroalimentaire. Au-delà des collaborations entre le secteur productif et académique qui doivent être plus vertueuses et porteuses de dynamiques d'apprentissage (que des instituts comme l'Institut de Technologie Alimentaire ambitionnent), il est nécessaire, de faire coexister le problème de l'informel dans la création de capacités [MUL, 11] et de rendre plus accessible le problème du financement dans le secteur industriel (et plus largement dans l'économie entière). La résolution (ou tout du moins la prise en compte plus affirmée) de ces problèmes structurels est à la base de la constitution de futures dynamiques industrielles prospères dans un pays où les NTIC ont très fortement progressé et où les projets portés sur les créations de parcs industriels ou technologiques affluent.

Notre proposition porte enfin une dernière recommandation de nature conceptuelle : l'intérêt de la mise en place de politiques de S\&T au sens «large ». Au Sénégal, si peu d'efforts sont déployés autour de la $S \& T$, et plus particulièrement de la R\&D industrielle, les mesures sont par contre réelles dans la mise en place de politiques indirectement liées à la $\mathrm{S} \& \mathrm{~T}$ et qui portent tout leur sens dans l'accompagnement de cette dernière [FAG, 15]. Par exemple, sans politique d'apprentissage volontariste comme pour la constitution d'ISEP ou de réformes dans l'enseignement supérieur, le marché du travail ne peut répondre à la demande de connaissances, propice à la création de capacités technologiques. En résulte le fameux brain drain tant marqué dans les économies sub-sahariennes.

Les limites de notre étude portent sur le manque de données microéconomiques, qui auraient pu enrichir la portée et cohérence du cadre régional sur les dynamiques d'innovation locales (notamment sectorielles). C'est donc dans cette direction et méthodologie bottom-up que nous complèterons ces travaux, en s'assurant toujours que les politiques de S\&T ne sont pas un «luxe » pour les pays en développement ou en transition mais bien contributives de leur équilibre à long terme.

\section{Bibliographie}

[ALP, 10] ALPHA A, ROLLAND J.P., 2010, Etude sur la cohérence des politiques commerciales en Afrique de l'Ouest, Rapport final, AFD, GRET.

[ANN, 05] ANNABI N., CISSÉ F., COCKBURN J., DECALUWÉ B., 2005, Trade Liberalisation, Growth and Poverty in Senegal : a Dynamic Microsimulation CGE Model Analysis, Document de Travail du CEPII, $n^{\circ} 2005-$ 07, mai.

[AST, 18] ASTI, 2018, Fiche d'information sur la R\&D agricole, Décembre 2018, Document disponible sur https://www.asti.cgiar.org/sites/default/files/pdf/Senegal-Fr-Factsheet-2018.pdf 
[BAS, 17] BASKARAN A., MUCHIE M., 2017, System Divergence or Coherence: The Variations of Innovation System from the Local to the Global, in MUCHIE M., BASKARAN A., Sectoral innovaton systems in Africa, Africa World Press, 15-37.

[BEN, 17] BEN SLIMANE S., RAMADAN M., 2017, Le système national d'innovation dans les pays du Maghreb : entre faille structurelle et besoin de coordination et de gouvernance appropriées, Innovations, 53, 2, 105-127.

[BIK, 10] BIKOUE S.M., 2010, Industrialisation par substitution des importations en Afrique et compétitivité internationale : une revue critique, Chapitre 6, Repenser les économies africaines pour le développement, BAZIKA, JC et A.B. NACIRI. 2010, Dakar : Codesria, 254 pages.

[BOR, 13] BORRAS S., EDQUIST C., 2013, "The choice of innovation policy instruments", Technological forecasting and social change, 1513-1522.

[CAR, 97] CARLSSON B., JACOBSSON S., 1997, "In search of a useful technology policy - general lessons and key issues for policy makers", in CARLSSON B., Technological systems and Industrial Dynamics. Kluwer Press, Boston, 299-315.

[CAS, 10] CASADELLA V., 2010, « Le SNI dans les PED », Editions Universitaires Européennes, 390 p.,

[CAS, 15] CASADELLA V., LIU Z., UZUNIDIS D., 2015, Développement économique et capacités d'innovation dans la mondialisation, ISTE Editions.

[CAS, 17] CASADELLA V., TAHI S., 2017, "Capacities and Innovation Policies in the Less Developed Countries: Lessons from the Case of Senegal", Innovations, vol. no 53, no. 2, 2017, p. 13-39.

[CHA, 18] CHAMINADE C., LUNDVALL B.A., HANEEF S., 2018, Advanced Introduction to National Innovation Systems, Elgar Publishing.

[DOG, 09] DOGSON M., 2009, "Asia's national innovation systems: institutional adaptability and rigidity in the face of global innovation challenges", Asia Pacific Journal of Management, 26 (3), 589-609.

[EDQ, 04] EDQUIST C., 2004, "Systems of Innovation: Perspectives and Challenges" in FAGERBERG J., MOWERY J., NELSON R., Oxford Handbook of Innovation, Oxford: Oxford University Press, 181-208

[FAG, 15] FAGERBERG J., 2015, "Innovation policy, national innovation systems and economic performance : in search of the useful theoretical framework", EU-SPRI conference "Science and innovation policy: Dynamics, Challenges, Responsibility and Practice”, Manchester, UK, 18-20 June, 2014.

[FAU, 19] FAURE G., KNIERIM A., KOUTSOURIS A., NDAH H.T., AUDOUIN S., ZAROKOSTA E., WIELINGA E., TRIOMPHE B., MATHE S., TEMPLE L., HEANUE K., 2019, How to strengthen innovation support services in agriculture with regard to multi-sakeholder approaches Journal of Innovation Economics and Management (28).145-169.

[FMI, 17]FMI, 2017, Rapport de 2016, Disponible $\quad$ sur https://www.imf.org/external/pubs/ft/ar/2016/eng/pdf/ar16_eng.pdf

[FREE, 87] FREEMAN, C.,1987, Technology and Economic Performance: Lessons from Japan, London,Pinter.

[GOU, 15] GOULET F., COMPAGNONGE C., LABERTHE P., 2015, Émergence des conseillers privés. De nouvelles interrogations pour la recherche, In Compagnone C., Goulet F., Labarthe P. (Eds.), Conseil privé en agriculture. Acteurs, pratiques, marché. Dijon, Educagri Editions;Versailles, Quae, 201-216.

[GOU, 18] GOUVERNEMENT DU SENEGAL, 2018, Plan Sénégal Emergent, Disponible sur https://www.unpage.org/files/public/plan_senegal_emergent.pdf

[HUG, 01] HUGON P., 2001, Analyse comparative des processus d'intégration économique régionale, Ministère des affaires étrangères, CERED.

[JAC, 93] JACQUEMOT P., RAFFINOT M., 1993, La nouvelle politique économique en Afrique, EDICEF/AUPELF, Universités francophones.

[LUN, 05] LUNDVALL B.A., BORRAS S., 2005, Science technology and innovation policy, in Oxford book of innovation, 599-631.

[OM, 94] OMAN C.,1994, Globalisation et régionalisation : quels enjeux pour les pays en développement, OCDE, Paris

[MAN, 18] MANSOOR A., ISSOUFOU S., 2018, Examen de la structure industrielle au Sénégal, in Fond Monétaire International, La course à la prochaine frontière de revenu, Comment le Sénégal et les autres pays à faible revenu peuvent franchir la ligne d'arrivée, IMF Press, p.43-61 
[MAR, 12] MARTIN, B., 2012, The evolution of science policy and innovation studies, Research Policy, 41, 12191239.

[MIN, 18] MINKOUA NZI J.R., TEMPLE L., 2018, L'offre de recherche converge-t-elle vers les besoins du secteur agro-alimentaire au Cameroun ? Une analyse par la bibliographie, Cahier agriculture 27, (25008).

[MUC, 03] MUCHIE M., GAMMERLTOFT P., LUNDVALL B., 2003, Putting Africa First: the making of African Innovation Systems, Aalborg University Press. Danemark.

[MUL, 11] MULLER, J., 2011, Making Ends Meet: Local socio-technological transformations in the South: based on case studies from Tanzania. Aalborg: Department of Development and Planning, Aalborg University.

[ROD, 10] RODRIK D., 2010, 'Diagnostics before prescription', The Journal of Economic Perspectives, 24(3), 33-44

[UNE, 15] UNESCO, 2015, Rapport de l'UNESCO sur la science, Editions UNESCO, 2016. 